\title{
A wandering brain reduces pain?
}

Activation of the default-mode network (DMN) - brain areas that are active during wakeful rest and the activity of which decreases during task performance - has been proposed to reflect 'mind-wandering. Karen Davis and her trainees Aaron Kucyi and Tim Salomons now show that the DMN remains active during spontaneous mind-wandering away from pain, and that this activity is associated with structural and functional connectivity between the DMN and a key opiate-rich region of the pain-modulation system.

Although pain is a highly salient stimulus, the experience of pain varies from moment to moment and among individuals. Specific cognitive strategies can reduce pain experience, but the authors focused on spontaneously occurring pain reductions by investigating whether they could be linked to DMN activity - for example, through connections between DMN areas and pain-modulation areas.

Volunteers received painful stimuli while fixating on a cross on a screen in a functional MRI (fMRI) scanner. After each stimulus they indicated the extent to which their thoughts and feelings had been focused on the pain or on something else, and whether that 'something else' was an external distraction (such as a sound), a task-related thought (such as what answer to give) or nothing in particular (that is, mind-wandering).
Trials in which attention was directed towards pain (P trials) were associated with activation of pain- and salience-related brain areas and, as expected, with deactivation in the DMN. If attention was somewhere else (SE trials), some of the same pain- and salience-related areas showed weaker activation, and there was no DMN deactivation. The difference between DMN activation in $P$ trials versus SE trials correlated with the degree to which the volunteers had attributed their 'something else' thought to mindwandering, suggesting that greater DMN activation during pain was associated with a tendency to mindwander away from pain.

SE trials were also associated with increased functional connectivity between DMN areas (including the medial prefrontal cortex (mPFC)) and the periaqueductal grey (PAG) - an antinociceptive area - during pain. Moreover, an individual's proportion of SE trials over P trials correlated both with structural connectivity between the mPFC and PAG and with the variability in resting-state functional connectivity between these areas. In other words, people who had a low intrinsic attention to pain had greater structural and more dynamic functional mPFC-PAG connectivity.
This study could not correlate actual pain ratings with $\mathrm{DMN}$ activity, as such ratings would have directed attention to the pain stimulus. Nevertheless, its findings suggest that sustained DMN activity during a painful stimulus may influence activity in pain-reducing areas such as the PAG.

Leonie Welberg

ORIGINAL RESEARCH PAPER Kucyi, A.,

Salomons, T. V. \& Davis, K. D. Mind wandering away from pain dynamically engages

antinociceptive and default mode brain networks.

Proc. Natl Acad. Sci. USA http://dx.doi. org/10.1073/pnas.1312902110(2013) FURTHER READING Bushnell, M. C., Čeko, M. 8 Low, L. A. Cognitive and emotional control of pain and its disruption in chronic pain. Nature Rev. Neurosci. 14, 502-511 (2013)

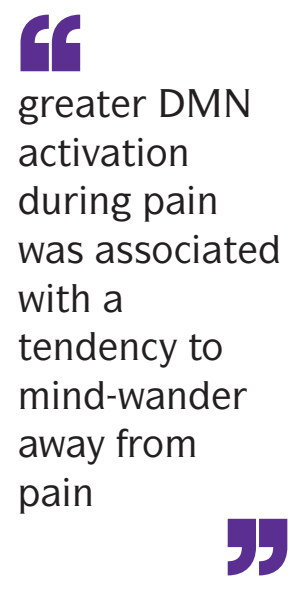

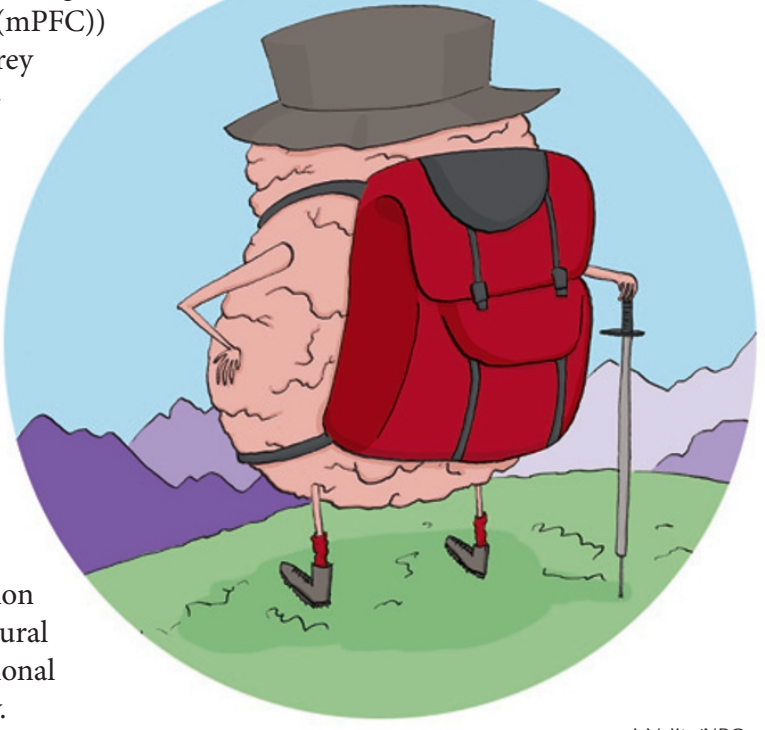

J. Vallis/NPG 